\title{
Phase-amplitude coupling in neuronal oscillator networks
}

\author{
Yuzhen Qin $\odot,{ }^{1, *}$ Tommaso Menara $\odot,{ }^{1}$ Danielle S. Bassett, ${ }^{2}$ and Fabio Pasqualetti ${ }^{1, \dagger}$ \\ ${ }^{1}$ Department of Mechanical Engineering, University of California, Riverside, California 92521, USA \\ ${ }^{2}$ Department of Physics and Astronomy, Department of Bioengineering, Department of Electrical \& Systems Engineering, \\ Department of Neurology, Department of Psychiatry, University of Pennsylvania, Philadelphia, Pennsylvania 19104, USA \\ and the Santa Fe Institute, Santa Fe, New Mexico 87501, USA
}

(Received 3 December 2020; accepted 1 June 2021; published 21 June 2021)

\begin{abstract}
Cross-frequency phase-amplitude coupling (PAC) describes the phenomenon where the power of a highfrequency oscillation evolves with the phase of a low-frequency one. It has been widely observed in the brain and linked to various brain functions. In this paper, we show that Stuart-Landau oscillators coupled in a nonlinear fashion can give rise to PAC in two commonly accepted architectures, namely, (1) a high-frequency neural oscillation driven by an external low-frequency input and (2) two interacting local oscillations with distinct, locally generated frequencies. We characterize the parameters that affect PAC behavior, thus providing insight on this phenomenon observed in neuronal networks. Inspired by some empirical studies, we further present an interconnection structure for brain regions wherein cross-region interactions are established only by lowfrequency oscillations. We then demonstrate that low-frequency phase synchrony can integrate high-frequency activities regulated by local PAC and control the direction of information flow between distant regions.
\end{abstract}

DOI: 10.1103/PhysRevResearch.3.023218

\section{INTRODUCTION}

Oscillatory activity at multiple frequency bands is widely observed in many natural, biological, and technological systems. Synchronization of oscillatory activity within the same frequency band has attracted extensive attention in past decades (e.g., see Ref. [1] and references therein). Yet, there is an emerging body of evidence revealing that brain oscillations in distinct frequency bands are also not independent, and they are instead found to interact through cross-frequency coupling $[2,3]$.

As one of the most prevalent representations of crossfrequency coupling, phase-amplitude coupling (PAC) occurs when the power (or amplitude) of a high-frequency rhythm is locked to (and often modulated by) the phase of a lowfrequency rhythm [4] [see Fig. 1(a)]. Brain signals recorded by various techniques, e.g., local field potential, electroencephalograph, and magnetoencephalograph, have revealed that PAC emerges in numerous brain regions, including auditory and prefrontal cortices [5], nucleus [6], and hippocampus [7], and plays a crucial role in motor functions [8] and cognitive processes such as working memory [9], attention [10], and learning [11].

Moreover, PAC usually does not emerge in the brain as an isolated phenomenon. In fact, regions exhibiting local

\footnotetext{
*yuzhenq@ucr.edu

†fabiopas@engr.ucr.edu
}

Published by the American Physical Society under the terms of the Creative Commons Attribution 4.0 International license. Further distribution of this work must maintain attribution to the author(s) and the published article's title, journal citation, and DOI.
PAC may also be phase-coupled in low-frequency bands. The combination of cross-region phase synchronization and local cross-frequency PAC can thus effectively integrate local information in distant brain regions (which is processed and regulated by high-frequency oscillations [4]). For instance, correlated $\gamma$ rhythms $(>30 \mathrm{~Hz})$ between the cortex and the striatum are typically established by cross-regional phase synchronization in the $\theta$ band $(4-8 \mathrm{~Hz})$ and $\theta-\gamma$ PAC that happen concurrently [12].

Existing work studies synchronization and PAC separately, yet these two phenomena often occur simultaneously and are generally interdependent. In the context of synchronization, there is a vast amount of literature on the analysis of phase-reduced models such as Kuramoto oscillators [13-17]. Previous studies have investigated synchronization under the influence of many different factors including time delays [18], noise $[19,20]$, and network symmetries [21]. Some work is also committed to the modeling of neuronal behaviors (e.g., see Refs. [22-24]). In the context of PAC, most research efforts are directed toward the description and analysis of this phenomenon in brain networks. Many of the existing models focus on the microscopic scale [25,26], but they fail to capture the behavior of larger neural populations. Some attempts have been made to describe PAC at the population level with the aid of neural mass models [27-31]. In those microscopic and population-level models, two architectures have been accepted to generate PAC [32]: (1) A neural population oscillating at high frequency (fast) is modulated by an external low-frequency (slow) input [see Fig. 1(b)]; (2) two populations that, respectively, generate fast and slow oscillations interact with each other [see Fig. 1(c)]. Yet, a model capable of assimilating the aforementioned instances with largescale synchronization of multiple regions is still critically missing. 


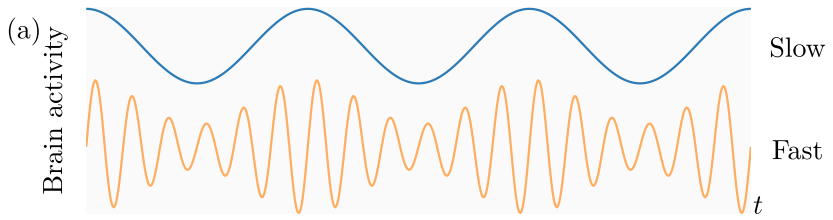

(1)

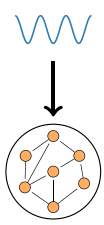

External input (c)

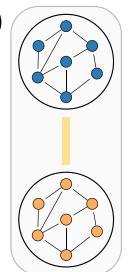

Local interaction (d)

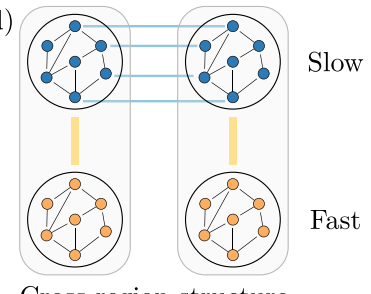

Cross-region structure
FIG. 1. An overview of PAC. (a) Illustration of PAC. (b), (c) Different architectures for the emergence of PAC: (b) A fast population with an external slow input (e.g., sensori stimulus); (c) Two interacting populations with distinct local oscillations (which also constitute a simplified brain region). (d) Cross-region interconnection structure.

In this paper, we employ the Stuart-Landau (SL) equation to model cross-frequency PAC and same-frequency synchrony by considering two coupling schemes. We show that SL oscillators with distinct frequencies coupled in a nonlinear fashion can exhibit PAC in both architectures depicted in Figs. 1(b)1(c). Our results reveal that specific parametric relationships modulate the emergence of PAC and its intensity, providing new insight on PAC behaviors in neuronal networks. For oscillators within the same frequency band, the standard linear coupling scheme for the SL model is considered, which characterizes same-frequency phase synchrony. Moreover, inspired by previous studies showing that low-frequency oscillations are better suited for establishing long-distance interactions than high-frequency ones [12,33], we put forth an interconnection structure [see Fig. 1(d)] in which crossregion interactions exist only between slow populations. This structure underlies the integration of cross-frequency coupling with same-frequency phase synchronization and constitutes a building block that can be easily scaled to form large networks. We then demonstrate the important role that long-distance same-frequency phase synchrony, together with regional PAC, plays in the coordination of high-frequency local activity and in information routing.

\section{OSCILLATOR MODEL}

We start by introducing the SL equation:

$$
\dot{z}=\left(\sigma+\mathrm{i} \omega-|z|^{2}\right) z
$$

where $z$ is a complex state $(z=x+\mathrm{i} y)$, with $\mathrm{i}=\sqrt{-1}$, and $\sigma$ and $\omega$ are real. The SL oscillator is also known as a Hopf oscillator, because, for $\sigma<0$, the model undergoes a subcritical Hopf bifurcation with $z=0$ being globally stable, and for $\sigma>0$, the model undergoes a supercritical Hopf bifurcation with $z$ converging to a limit cycle of radius $\sqrt{\sigma}$ and angular frequency $\omega$. The SL oscillator can equivalently be described in polar coordinates by letting $z=r e^{i \theta}$, where $r$ and $\theta$ are referred to as the amplitude and phase angle of the oscillator, respectively. While phase-only models such as the Kuramoto model have been widely used to study synchrony in brain oscillations, the SL model, like many other phase-amplitude models [34-36], can capture richer behaviors than phase-only ones $[37,38]$.

Many studies have shown that the dynamics of a network of neurons reduce to the SL equation (e.g., see Refs. [39,40]). Therefore, despite the apparent simplicity, the SL equation has been used to approximate neural behaviors at the population level (e.g., see Refs. [24,41,42]). Local synaptic coupling can synchronize the firing activity of neurons [32] and then bring the population from a stationary to an oscillatory regime by shifting the parameter $\sigma$ in Eq. (1) from negative to positive. The oscillation frequency mainly depends on the intrinsic decay time of inhibition in the population [43].

Inspired by the previous work, we use the SL equation given in Eq. (1) as a model system in this paper, aiming at providing insight on collective behaviors of neuronal networks. The simplicity of the SL model enables us to describe the dynamics of large networks and analyze how the parameters of the networks affect the collective behaviors across frequencies. The analysis leads to some theoretical hypotheses that may be tested in networks of real neuronal populations.

\section{EMERGENCE OF PAC BY EXOGENOUS INPUTS}

We first show how PAC can arise in a population oscillating at a high frequency subject to a slow exogenous input [i.e., the architecture depicted in Fig. 1(b)].

Consider the dynamics of the population with an input $u$, i.e.,

$$
\dot{z}_{\mathrm{f}}=\left(\delta_{\mathrm{f}}+\mathrm{i} \omega_{\mathrm{f}}-\left|z_{\mathrm{f}}\right|^{2}\right) z_{\mathrm{f}}+z_{\mathrm{f}} k_{I} u,
$$

where $z_{\mathrm{f}}=x_{\mathrm{f}}+\mathrm{i} y_{\mathrm{f}}$. We let $\delta_{\mathrm{f}}>0$ and $\omega_{\mathrm{f}}>0$ such that there is a fast intrinsic oscillation in this neuronal population. Such self-sustained, fast oscillations (e.g., $\gamma$-oscillations) have been observed in hippocampus [11], striatum [44], neocortex [45], and other brain areas.

The input $u$ can represent various sensory signals from sensory cortices $[32,46]$. A complex-valued $u$ brings changes to the fast self-sustained oscillation by shifting the parameters $\delta_{\mathrm{f}}$ and $\omega_{\mathrm{f}}$. The strength $k_{I}$ scales the influence of $u$ on the fast oscillation.

Although more general inputs are allowed, we consider $u$ a sinusoidal signal of amplitude (or strength) $k_{\mathrm{I}}$ for the purposes of simple illustration, i.e., $u=\sin \left(\omega_{\mathrm{I}} t\right)$. Consequently,

$$
\dot{z}_{\mathrm{f}}=\left(\delta_{\mathrm{f}}+k_{\mathrm{I}} \sin \left(\omega_{\mathrm{I}} t\right)+\mathrm{i} \omega_{\mathrm{f}}-\left|z_{\mathrm{f}}\right|^{2}\right) z_{\mathrm{f}} .
$$

Then, $\sigma$, which determines the types of bifurcation in Eq. (1), becomes time dependent, i.e., $\sigma(t)=\delta_{\mathrm{f}}+k_{\mathrm{I}} \sin \left(\omega_{\mathrm{I}} t\right)$. It can be seen that the fast amplitude $r_{\mathrm{f}}$ tends to converge to $\sqrt{\sigma(t)}$, which means that PAC should emerge.

Notice that, depending on how the fast oscillation behaves throughout the slow cycle, PAC can be categorized into continuous and intermittent [32]. In the former, the fast oscillation is constantly active, while in the latter, the fast oscillation only appears in a certain phase interval of the slow cycle. Our model is capable of capturing both categories of PAC, and the input magnitude determines which type will occur. We remark that PAC is different from beating, a well-known phenomenon in acoustic and mechanical systems, describing 

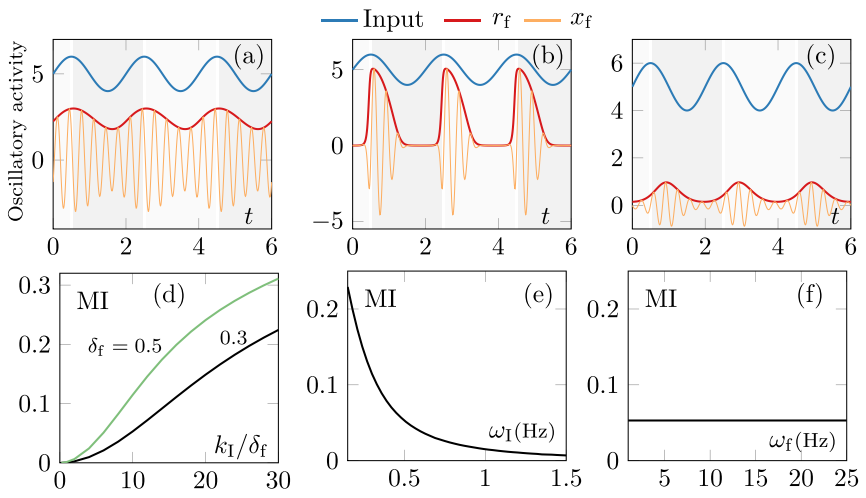

FIG. 2. PAC by exogenous inputs. (a), (b) A weaker input $\left(k_{\mathrm{I}}=\right.$ $3)$ yields continuous PAC, while a strong one $\left(k_{\mathrm{I}}=18\right)$ yields intermittent PAC, where $\delta_{\mathrm{f}}=6$ for both. (c) The $\delta_{\mathrm{f}}$ in (a) is reduced from 6 to 0.3 , and a noticeable phase lag appears between peaks (or valleys) of the fast amplitude oscillation and the slow phase oscillation. (d)-(f) The dependence of PAC intensity (quantified by the MI) on the input strength (d), the input frequency (e), and the fast frequency (f). (Parameters adopted in this figure: If not specified otherwise, $\delta_{\mathrm{f}}=0.3, k_{\mathrm{I}}=3 \omega_{f}=3 \mathrm{~Hz}$, and $\omega_{\mathrm{I}}=0.5 \mathrm{~Hz}$.)

the situation where the superposition of two signals with similar frequencies and amplitudes exhibits a periodic variation on the amplitude envelope. Unlike PAC, there is no other signal modulating the variation of the amplitude envelope [47].

For a weak input, where $k_{\mathrm{I}}<\delta_{\mathrm{f}}, \sigma(t)$ is always positive and the fast population remains in the oscillatory regime, which implies that continuous PAC arises [see Fig. 2(a)]. Conversely, for a strong input, where $k_{\mathrm{I}}>\delta_{\mathrm{f}}, \sigma(t)$ can be negative in some interval of the slow cycle. In these intervals, the fast population is driven out of the oscillatory regime. Thus, $r_{\mathrm{f}}$ tends toward 0 , and the fast oscillation may disappear. We illustrate intermittent PAC for large $k_{\mathrm{I}}$ in Fig. 2(b).

One can observe from Figs. 2(a) and 2(b) that the peaks and valleys of the fast amplitude oscillation appear almost simultaneously as those of the slow input oscillation, respectively. However, this situation does not always happen, and it highly depends on $\delta_{\mathrm{f}}$ in Eq. (2). As pointed out in Ref. [48], since $\sigma$ in Eq. (1) determines the convergence rate to the limit cycle, we then reason that the rate of $r_{\mathrm{f}}$ tracking $\sqrt{\sigma(t)}$ is also positively correlated with $\delta_{\mathrm{f}}$. Therefore, a larger $\delta_{\mathrm{f}}$ means a smaller phase lag. Figure 2(c) demonstrates that a noticeable phase lag appears for a smaller $\delta_{\mathrm{f}}$ in comparison with Fig. 2(a).

After revealing the emergence of PAC, we investigate how this phenomenon depends on the model parameters. More precisely, we utilize the Modulation Index (MI) [49] to measure PAC intensity (see its definition and details for its computation in the Appendix). As we have reasoned that the fast amplitude $r_{\mathrm{f}}$ tends to track $\sqrt{\sigma(t)}=\sqrt{\delta_{\mathrm{f}}} \cdot \sqrt{1+k_{\mathrm{I}} / \delta_{\mathrm{f}} \sin \left(\omega_{\mathrm{I}} t\right)}$, it is clear that a larger ratio $k_{\mathrm{I}} / \delta_{\mathrm{f}}$ implies larger fluctuations in $r_{\mathrm{f}}$ than a weaker one. That is, the PAC intensity increases with $k_{\mathrm{I}} / \delta_{\mathrm{f}}$ [see Fig. 2(d)]. Additionally, for a fixed a ratio $k_{\mathrm{I}} / \delta_{\mathrm{f}}$, variations of $\delta_{\mathrm{f}}$ impact the fluctuations of $r_{\mathrm{f}}$ : A larger $\delta_{\mathrm{f}}$ implies more intense PAC [see Fig. 2(d)].

The input frequency $\omega_{\mathrm{I}}$, which is called modulating frequency, also has a profound impact on the PAC intensity. As $\sigma(t)=\delta_{\mathrm{f}}+k_{\mathrm{I}} \sin \left(\omega_{\mathrm{I}} t\right)$, a smaller $\omega_{\mathrm{I}}$ means a more slowly varying $\sigma(t)$, which is easier for the fast amplitude $r_{\mathrm{f}}$ to track. Figure 2(e) shows that the PAC intensity decreases as $\omega_{\mathrm{I}}$ increases. By contrast, the fast frequency $\omega_{\mathrm{f}}$ has little influence on the PAC intensity [see Fig. 2(f)].

So far we have focused on the emergence of PAC in the architecture shown in Fig. 1(b). That is, a slowly oscillating input to the fast population modulates the fast amplitude. However, some in vitro studies have shown that besides highfrequency rhythms, low-frequency ones can also be generated locally in a single brain region [50,51]. These heterogeneous rhythms then interact as in the architecture depicted in Fig. 1(c). We now turn to the study of this architecture.

\section{PAC BY LOCAL INTERACTIONS: SHAPING OF A BRAIN REGION}

As depicted in Fig. 1(c), a fast population may locally interact with a slow one. The two population dynamics are

$$
\begin{aligned}
& \dot{z}_{\mathrm{s}}=\left(\delta_{\mathrm{s}}+\mathrm{i} v_{\mathrm{s}}-\left|z_{\mathrm{s}}\right|^{2}\right) z_{\mathrm{s}}+z_{s} f\left(z_{\mathrm{f}}\right), \\
& \dot{z}_{\mathrm{f}}=\left(\delta_{\mathrm{f}}+\mathrm{i} v_{\mathrm{f}}-\left|z_{\mathrm{f}}\right|^{2}\right) z_{\mathrm{f}}+z_{f} f\left(z_{\mathrm{s}}\right),
\end{aligned}
$$

where $z_{\mathrm{s}}=x_{s}+\mathrm{i} y_{\mathrm{s}}, z_{\mathrm{f}}=x_{\mathrm{f}}+\mathrm{i} y_{\mathrm{f}}$, and $\delta_{\mathrm{s}}, \delta_{\mathrm{f}}>0$. The oscillators' natural frequencies are determined by $v_{\mathrm{s}}$ and $v_{\mathrm{f}}$, with $v_{\mathrm{f}}>v_{\mathrm{s}}$. The complex function $f(\cdot)$ describes how the two populations are interconnected. If the connection is unidirectional from the slow population to the fast one (a case studied in Ref. [52]), i.e., $f\left(z_{\mathrm{f}}\right)=0$, then Eq. (4) reduces to Eq. (2) with input $u=f\left(z_{\mathrm{s}}\right)$. In this case, our analysis above concludes that PAC emerges if $f\left(z_{\mathrm{s}}\right)$ is a function of the slow phase.

We next turn our attention to the more interesting case wherein the interaction between the two populations is bidirectional. In this paper, we simply consider $f(z)=k z$, and it is worth mentioning that our analysis below applies to other interconnection functions. Then, Eq. (3) and Eq. (4) become

$$
\begin{aligned}
& \dot{z}_{\mathrm{s}}=\left[\left(\delta_{\mathrm{s}}+k x_{\mathrm{f}}\right)+\mathrm{i}\left(v_{\mathrm{s}}+k y_{\mathrm{f}}\right)-\left|z_{\mathrm{s}}\right|^{2}\right] z_{\mathrm{s}}, \\
& \dot{z}_{\mathrm{f}}=\left[\left(\delta_{\mathrm{f}}+k x_{\mathrm{s}}\right)+\mathrm{i}\left(v_{\mathrm{f}}+k y_{\mathrm{s}}\right)-\left|z_{\mathrm{f}}\right|^{2}\right] z_{\mathrm{f}} .
\end{aligned}
$$

In polar coordinates, $z_{\mathrm{s}}=r_{\mathrm{s}} e^{\mathrm{i} \theta_{\mathrm{s}}}$ and $z_{\mathrm{f}}=r_{\mathrm{f}} e^{\mathrm{i} \theta_{\mathrm{f}}}$. It can be observed that the terms $\sigma$ and $\omega$, which determine the amplitude and phase in the slow and fast population in Eq. (1), read as $\sigma_{\mathrm{s}}=\delta_{\mathrm{s}}+k x_{\mathrm{f}}, \omega_{\mathrm{s}}=v_{\mathrm{s}}+k y_{\mathrm{f}}$, and $\sigma_{\mathrm{f}}=\delta_{\mathrm{f}}+k x_{\mathrm{s}}, \omega_{\mathrm{f}}=v_{\mathrm{f}}+$ $k y_{\mathrm{s}}$, respectively. Interestingly, the amplitudes and phases of the two populations become dependent on one another due to the interconnection. Notice that such dependence is asymmetric due to the frequency difference.

We find that the slow oscillation remains relatively independent from the fast one. Since $v_{\mathrm{f}}>v_{\mathrm{s}}$, one can see that $z_{\mathrm{f}}$ oscillates faster than the $z_{\mathrm{s}}$. Applying averaging techniques developed in Ref. [53] to the dynamics in Eq. (5), we obtain the approximate guiding system

$$
\dot{\hat{z}}_{\mathrm{s}}=\left(\delta_{\mathrm{s}}+\mathrm{i} v_{\mathrm{s}}-\left|\hat{z}_{\mathrm{s}}\right|^{2}\right) \hat{z}_{\mathrm{s}},
$$

which corresponds to the dynamics of the decoupled slow population. The solutions to Eq. (5) and the guiding system satisfy

$$
\left\|z_{\mathrm{s}}(t)-\hat{z}_{\mathrm{s}}(t)\right\| \leqslant c v_{\mathrm{s}} / v_{f}
$$



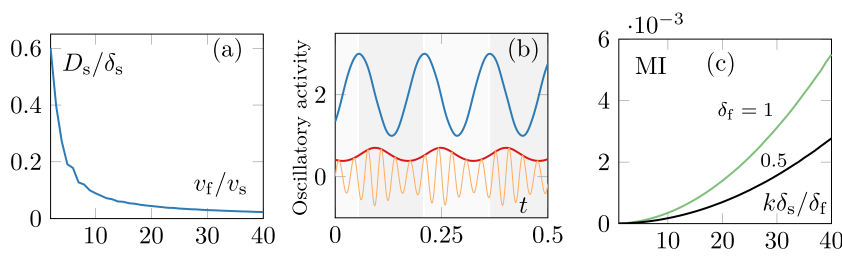

FIG. 3. PAC by local interactions. (a) $D_{s} / \delta_{\mathrm{s}}$ with $D_{s}=$ $\sup _{t}\left\|z_{\mathrm{s}}(t)-\hat{z}_{\mathrm{s}}(t)\right\|$ measures the derivation of $z_{\mathrm{s}}(t)$ from the solution of its decoupled counterpart. As $v_{\mathrm{f}} / v_{\mathrm{s}}$ increases, the derivation shrinks dramatically $\left(k=5, \delta_{\mathrm{f}}=0.3\right)$. (b) PAC emerges by local interaction between two populations as in Fig. 1 (c), where the slow frequency is $6.5 \mathrm{~Hz}$ ( $\theta$ band), and the fast one is $30 \mathrm{~Hz}$ ( $\gamma$ band). (c) PAC intensity (measured by the MI) increases with $k \delta_{\mathrm{s}} / \delta_{\mathrm{f}}$. Given a fixed ratio $k \delta_{\mathrm{s}} / \delta_{\mathrm{f}}$, PAC becomes more intense for larger $\delta_{\mathrm{f}}$.

for some positive $c$. Loosely speaking, the slow trajectory $z_{\mathrm{s}}(t)$ fluctuates in the neighborhood of the trajectory of its decoupled counterpart $\hat{z}_{\mathrm{s}}(t)$. Such fluctuations decrease as the two frequencies separate [see Fig. 3(a)].

In contrast, Eq. (6) can be rewritten as

$$
\dot{z}_{\mathrm{f}}=\left[\left(\delta_{\mathrm{f}}+k r_{\mathrm{s}} \cos \theta_{\mathrm{s}}\right)+\mathrm{i}\left(v_{\mathrm{f}}+r_{\mathrm{s}} \sin \theta_{\mathrm{s}}\right)-\left|z_{\mathrm{f}}\right|^{2}\right] z_{\mathrm{f}}
$$

Similar to our earlier analysis, the fast amplitude $r_{\mathrm{f}}$ tends to track $\sqrt{\delta_{\mathrm{f}}+k r_{\mathrm{s}} \cos \theta_{\mathrm{s}}}$, which implies PAC.

Let the fast population in Fig. 1(c) oscillate in the $\gamma$ band and the slow population oscillate in the $\theta$ band (a choice compatible with empirical observations). Figure 3(b) shows that PAC emerges from the interaction of these two populations. The PAC intensity here also depends on the slow amplitude $r_{\mathrm{s}}$, which is determined by $\delta_{\mathrm{s}}$. Note that the connection strength $k$ also affects PAC intensity. Therefore, one can infer that the PAC becomes more intense as $k \delta_{\mathrm{s}} / \delta_{\mathrm{f}}$ increases [see Fig. 3(c)]. Similar to the previous section, a larger $\delta_{\mathrm{f}}$ also yields more intense PAC.

So far a single oscillator has been employed to capture the dynamics of oscillations in each frequency band. In practice, each frequency band may consist of a number of similar but heterogeneous oscillators, forming slow and fast clusters [see Fig. 4(a)]. The oscillators are diffusively connected within each cluster, and the cross-cluster (or cross-frequency) interconnections are in the same nonlinear fashion as considered in Eq. (5) and Eq. (6). Then, their dynamics are governed by

$$
\begin{aligned}
\dot{z}_{\mathrm{s}}^{i}= & \left(\delta_{\mathrm{s}}^{i}+\mathrm{i} v_{\mathrm{s}}^{i}-\left|z_{\mathrm{s}}^{i}\right|^{2}\right) z_{\mathrm{s}}^{i} \\
& +\frac{k_{\mathrm{s}}}{n} \sum_{j=1}^{n} a_{i j}\left(z_{\mathrm{s}}^{j}-z_{\mathrm{s}}^{i}\right)+\frac{k}{m} z_{\mathrm{s}}^{i} \sum_{p=1}^{m} c_{i p} z_{\mathrm{f}}^{p}, \\
\dot{z}_{\mathrm{f}}^{p}= & \left(\delta_{\mathrm{f}}^{p}+\mathrm{i} v_{\mathrm{f}}^{p}-\left|z_{\mathrm{f}}^{p}\right|^{2}\right) z_{\mathrm{f}}^{p} \\
& +\frac{k_{\mathrm{f}}}{m} \sum_{q=1}^{m} b_{p q}\left(z_{\mathrm{f}}^{q}-z_{\mathrm{f}}^{p}\right)+\frac{k}{n} z_{\mathrm{f}}^{p} \sum_{i=1}^{n} c_{i p} z_{\mathrm{s}}^{i},
\end{aligned}
$$

where we have assumed that there are $n$ and $m$ oscillators in the slow and fast clusters, respectively. The matrix $A=\left[a_{i j}\right] \in$ $\mathbb{R}^{n \times n}$ is the adjacency matrix of the slow cluster, where there is an undirected connection between the $i$ th and $j$ th oscillators if $a_{i j}>0$. Likewise, $B=\left[b_{p q}\right] \in \mathbb{R}^{n \times n}$ is the adjacency matrix of the fast cluster. The matrix $C=\left[c_{i p}\right] \in \mathbb{R}^{n \times m}$ describes the (a)

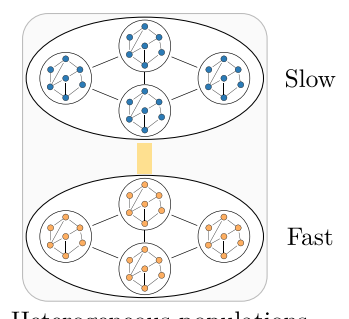

Heterogeneous populations (b)

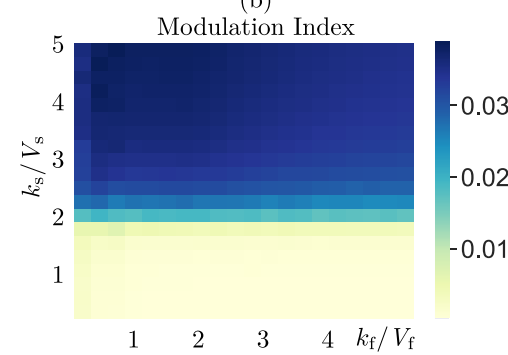

FIG. 4. General structure for a brain region. (a) Heterogeneous oscillators constitute each frequency band. (b) The dependence of the PAC intensity (quantified by the MI) on the intracluster coupling strengths $k_{\mathrm{s}}$ and $k_{\mathrm{f}}$. For simplicity, we consider $n=m=20$, and the within-cluster connections and the cross-cluster interconnections are both assumed to be all-to-all. Parameters $\delta_{\mathrm{s}}^{i}, \delta_{\mathrm{f}}^{p}, v_{\mathrm{s}}^{i}$, and $v_{\mathrm{f}}^{p}$ are randomly drawn from normal distributions $\mathcal{N}\left(\delta_{\mathrm{s}}, \Delta_{\mathrm{s}}\right), \mathcal{N}\left(\delta_{\mathrm{f}}, \Delta_{f}\right)$, $\mathcal{N}\left(v_{\mathrm{s}}, V_{\mathrm{s}}\right)$, and $\mathcal{N}\left(v_{\mathrm{f}}, V_{\mathrm{f}}\right)$, respectively. Each cluster is considered to consist of 20 oscillators, and $K=1,\left(\delta_{\mathrm{s}}, \Delta_{\mathrm{s}}\right)=(8,0.8),\left(\delta_{\mathrm{f}}, \Delta_{\mathrm{f}}\right)=$ $(1,0.1),\left(v_{\mathrm{s}}, V_{\mathrm{s}}\right)=(4,0.4)$, and $\left(v_{\mathrm{f}}, V_{\mathrm{f}}\right)=(40,2)$. For each pair of $k_{\mathrm{s}}$ and $k_{\mathrm{f}}$, the MI value in the figure is the mean for 1000 random trials.

cross-frequency interconnections, i.e., there is an undirected interconnection between the $i$ th oscillator in the slow cluster and the $p$ th oscillator in the fast cluster if $c_{i p}>0$. Here $k_{\mathrm{s}}$ and $k_{\mathrm{f}}$ are the coupling strengths within the slow and fast cluster, respectively, and $k$ represents the cross-frequency coupling strength.

Phase synchrony may affect the intensity of PAC between the slow and the fast clusters. Surprisingly, for a fixed intercluster connection $k$, Fig. 4(b) shows that the intensity of PAC mainly depends on the $k_{\mathrm{s}}$. In other words, the synchrony level of the modulating (slow) cluster, determined by the coupling strength of the slow cluster $k_{\mathrm{s}}$, plays a dominant role in controlling the PAC intensity. In contrast, the synchrony in the modulated cluster is negligible.

We put forth that the architecture depicted in Fig. 1(c) [or a more general version Fig. 4(a)] constitutes the basic organization of a brain region containing oscillations in two frequency bands. This architecture can be easily extended to account for situations in which more than two frequency bands coexist in a single brain region.

Because numerous brain functions require synchronous activation of different brain regions, we now extend the singleregion architecture discussed above [see Fig. 1(c)] to multiple coupled regions.

\section{PHASE SYNCHRONY GOVERNS PAC ACROSS DISTANT REGIONS}

As a building block for more complex network structures, we consider a two-region clique as depicted in Fig. 1(d), wherein interaction across brain regions is established by oscillations in the low-frequency range. Then, we show how cross-region phase synchrony contributes to integrate local high-frequency activities across long distances and to control the direction of information flow between regions. 

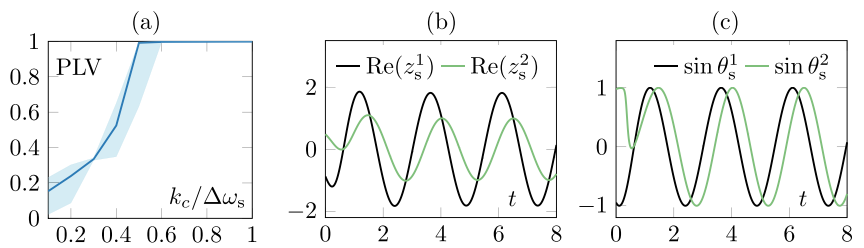

FIG. 5. Roles of low-frequency phase synchrony. (a) PLV of the fast amplitudes in the two regions against the ratio $K_{c} / \Delta \omega_{\mathrm{s}}$. The line indicated the mean of 1000 random realizations contained in the shaded area. It can be inferred that the fast populations becomes more correlated as the synchrony of the slow oscillations increases. (b), (c) Lead-lag relationship between the two signals: $z_{\mathrm{s}}^{1}$ precedes $z_{\mathrm{s}}^{2}$ if $v_{\mathrm{s}}^{1}>v_{\mathrm{s}}^{2}$ and vice versa.

Under the structure depicted in Fig. 1(d), the dynamics of the neural populations become

$$
\begin{gathered}
\dot{z}_{\mathrm{s}}^{p}=\left(\delta_{\mathrm{s}}^{p}+\mathrm{i} v_{\mathrm{s}}^{p}-\left|z_{\mathrm{s}}^{p}\right|^{2}\right) z_{\mathrm{s}}+k^{p} z_{\mathrm{s}}^{p} z_{\mathrm{f}}^{p}+k_{c}\left(z_{\mathrm{s}}^{-p}-z_{\mathrm{s}}^{p}\right), \\
\dot{z}_{\mathrm{f}}^{p}=\left(\delta_{\mathrm{f}}^{p}+\mathrm{i} v_{\mathrm{f}}^{p}-\left|z_{\mathrm{f}}^{p}\right|^{2}\right) z_{\mathrm{f}}+k^{p} z_{\mathrm{f}}^{p} z_{\mathrm{s}}^{p},
\end{gathered}
$$

where the superscript $p \in\{1,2\}$ is the region index $(-p=2$ if $p=1$ and $-p=1$ otherwise). Notice that the cross-region connection within the same frequency band is diffusive, which permits synchrony of slow oscillations. Here, $k^{p}$ and $k_{c}$ are the within- and cross-region connection strengths, respectively.

We find that the cross-region connection strength $k_{c}$ determines the phase synchrony between the two slow populations. One can reason that if the slow phases are synchronized, the fast amplitudes also behave coherently, provided there is local PAC in each region. Let $\bar{r}_{\mathrm{f}}^{1}$ and $\bar{r}_{\mathrm{f}}^{2}$ be the average amplitudes in the two regions. To measure the correlation of these two amplitudes, we calculate the phase-locking value (PLV) [49]. A large PLV implies that the two amplitudes are highly correlated (see the definition of computation of PLV in the Appendix). Let $\Delta \omega_{\mathrm{s}}=\left|v_{\mathrm{s}}^{1}-v_{\mathrm{s}}^{2}\right|$ be the natural frequency of the two slow populations. The ratio $k_{c} / \Delta \omega_{\mathrm{s}}$ determines the synchrony of the slow populations. Figure 5(a) shows that the PLV increases with the ratio $k_{c} / \Delta \omega_{s}$. Phase synchronization in the low-frequency range coordinates local high-frequency activities regulated by PAC, which is consistent with empirical studies such as Ref. [12].

Finally, we put forth that the considered model may shed new light on the directionality of information flow across brain regions. Specifically, we show how natural frequency differences affect the lead-lag relationship of oscillatory rhythms between brain regions. It suffices to investigate the lead-lag direction in the low-frequency range, as we already know that slow oscillations govern communications across regions. Moreover, within each region the slow population remains relatively independent from the fast one. Thus it is sufficient to study the two diffusively coupled slow populations in Fig. 1(d), whose dynamics are approximately

$$
\dot{z}_{\mathrm{s}}^{p}=\left(\delta_{\mathrm{s}}^{p}+\mathrm{i} v_{\mathrm{s}}^{p}-\left|z_{\mathrm{s}}^{p}\right|^{2}\right) z_{\mathrm{s}}+k_{c}\left(z_{\mathrm{s}}^{-p}-z_{\mathrm{s}}^{p}\right),
$$

where $p=1,2$, and $-p=2$ if $p=1,-p=1$ if $p=2$. In polar coordinates, $z_{\mathrm{s}}^{p}=r_{\mathrm{s}}^{p} e^{i \theta_{s}^{p}}$, and Eq. (9) can be rewritten as

$$
\begin{aligned}
& \dot{r}_{\mathrm{s}}^{p}=\left[\delta_{\mathrm{s}}^{p}-\left(r_{\mathrm{s}}^{p}\right)^{2}\right] r_{\mathrm{s}}^{p}+k_{c}\left[r_{\mathrm{s}}^{-p} \cos \left(\theta_{\mathrm{s}}^{-p}-\theta_{\mathrm{s}}^{p}\right)-r_{\mathrm{s}}^{-p}\right], \\
& \dot{\theta}_{\mathrm{s}}^{p}=v_{\mathrm{s}}^{p}+k_{c} \frac{r_{\mathrm{s}}^{-p}}{r_{\mathrm{s}}^{p}} \sin \left(\theta_{\mathrm{s}}^{-p}-\theta_{\mathrm{s}}^{p}\right) .
\end{aligned}
$$

For a strong connection $k_{c}$, the system converges to a solution with synchronized frequencies and constant but different amplitudes, i.e., $\dot{\theta}_{\mathrm{s}}^{1}-\dot{\theta}_{\mathrm{s}}^{2}=0, \dot{r}_{\mathrm{s}}^{1}=0$, and $\dot{r}_{\mathrm{s}}^{2}=0$. Letting $\theta_{\mathrm{s}}^{1}-\theta_{\mathrm{s}}^{2}=c_{12}$ yields

$$
v_{\mathrm{s}}^{1}-v_{\mathrm{s}}^{2}-k_{c}\left(\frac{r_{\mathrm{s}}^{2}}{r_{\mathrm{s}}^{1}}+\frac{r_{\mathrm{s}}^{1}}{r_{\mathrm{s}}^{2}}\right) \sin c_{12}=0,
$$

which has a solution

$$
c_{12}=\arcsin \left(\frac{v_{\mathrm{s}}^{1}-v_{\mathrm{s}}^{2}}{k_{c} \lambda}\right),
$$

with $\lambda r_{\mathrm{s}}^{2} / r_{\mathrm{s}}^{1}+r_{\mathrm{s}}^{1} / r_{\mathrm{s}}^{2}>0$. Note that we do not discuss the other solution $c_{12}=\pi-\arcsin \left(\frac{v_{s}^{1}-v_{s}^{2}}{k_{c} \lambda}\right)$ since it is unstable. The system converges to a solution wherein the phase $\theta_{\mathrm{s}}^{2}$ lags behind (resp. leads) $\theta_{\mathrm{s}}^{1}$ if $v_{\mathrm{s}}^{1}>v_{\mathrm{s}}^{2}\left(\right.$ resp. $v_{\mathrm{s}}^{1}<v_{\mathrm{s}}^{2}$ ), which we illustrate in Figures. 5(b)-5(c). Suppose $v_{\mathrm{s}}^{1}>v_{\mathrm{s}}^{2}$, then we have that

$$
z_{\mathrm{s}}^{2}(t)=r_{\mathrm{s}}^{2} \cos \left[\theta_{\mathrm{s}}^{2}(t)\right]+\mathrm{i} \sin \left[\theta_{\mathrm{s}}^{2}(t)\right]=\frac{r_{\mathrm{s}}^{2}}{r_{\mathrm{s}}^{1}} z_{\mathrm{s}}^{1}\left(t-\frac{c_{12}}{\omega}\right),
$$

with $\omega$ being the synchronized frequency, which means that $z_{\mathrm{s}}^{2}$ lags behind $z_{\mathrm{s}}^{1}$ by $\tau=c_{12} / \omega$. This behavior may suggest that the former acts as the information sender, and the latter acts as the receiver, since phase lead-lag relationship is widely used in previous studies to infer the directionality of the information flow (e.g., see Refs. [54,55]).

To summarize, we postulate that synchronization supports effective communication, and that the natural frequency differences determine the information flow directionality. We conjecture that the brain is capable of controlling this directionality effectively by manipulating the natural frequencies of neural populations. Further experimental studies are needed to investigate these conjectures. We remark that our findings provide some useful insights regarding the inference of directionality in information flow. As low-frequency oscillations are much more engaged in cross-region communication than the high-frequency counterparts, the latter may be filtered out from recorded signals when analyzing information flow across distant regions.

\section{CONCLUDING REMARKS}

In this paper, we have focused on studying cross-frequency phase-amplitude coupling in neuronal networks. With the aid of the SL equation and a nonlinear coupling scheme, we demonstrate the parametric relationships that modulate PAC intensity. These relationships point to hypotheses that could be tested for real neuronal networks. Different from exiting models that are intricate, the considered model is clean and simple enough to allow theoretical insights. Further, we have provided a cross-region structure for interactions of multiple brain regions, which enable the study of PAC and phase synchronization simultaneously. We believe that the combination 
of this interconnection structure and the the SL model may also pave a way to study other cross-frequency coupling apart from PAC, same-frequency phase synchrony, and their interplay.

The brain is a complex network. How different regions are integrated in numerous cognitive processes may depend on brain network structure. Our work can be further extended to study the important role of network structure in affecting cross-frequency coupling, phase synchronization, and, in turn, the cognitive performance such as working memory, attention, and learning.

\section{ACKNOWLEDGMENTS}

This work was supported in part by awards Army Research Office (ARO), Award No. W911NF-18-1-0213 and National Science Foundation (NSF), Award No. NCS-FO-1926829.

\section{APPENDIX}

\section{Modulation Index}

Since its introduction, the MI serves as a popular measure to quantify the PAC intensity [49]. In the following, we summarize the steps to compute the MI.

We first derive the phase-amplitude distribution $P$ of two complex signals-a slow one, $z_{\mathrm{s}}(t)=x_{\mathrm{s}}(t)+\mathrm{i} y_{\mathrm{s}}(t)$, and fast one, $z_{\mathrm{f}}(t)=x_{\mathrm{f}}(t)+\mathrm{i} y_{\mathrm{f}}(t)$-as follows:

(1) We extract the time series of the phases of $z_{\mathrm{s}}(t)$. For each time instant $t$, the phase is $\theta_{\mathrm{s}}(t)$ in $z_{\mathrm{s}}(t)=r_{\mathrm{S}}(t) e^{\mathrm{i} \theta_{\mathrm{s}}(t)}$.

(2) Extract the time series of the amplitude envelope of $z_{\mathrm{f}}(t)$. Analogously, the amplitude at each time instant $t$ is $r_{\mathrm{f}}(t)$ in $z_{\mathrm{f}}(t)=r_{\mathrm{f}}(t) e^{\mathrm{i} \theta_{\mathrm{f}}(t)}$.

(3) Divide the phases $\theta_{\mathrm{s}}(t)$ into $n$ bins, where $n$ can be any positive number. We use $n=18$ in this paper, i.e., we bin the phase interval 0 to $360^{\circ}$ into 18 intervals of $20^{\circ}$. For each bin, we calculate the mean of $r_{\mathrm{f}}(t)$, denoted by $\left\langle r_{\mathrm{f}}\right\rangle_{i}$. Specifically, $\left\langle r_{\mathrm{f}}\right\rangle_{i}=\frac{1}{N} \sum_{j=1}^{N} r_{\mathrm{f}}\left(t_{j}\right)$, where $t_{j}$ are the sample points such that $\theta_{s}\left(t_{j}\right)$ belong to the $i$ th bin and $N$ is the number of such sample points.

(4) Normalize the mean amplitude in each bin by $P(j)=$ $\frac{\left\langle r_{\mathrm{f}}\right\rangle_{i}}{\sum_{j=1}^{n}\left\langle r_{\mathrm{f}}\right\rangle_{j}}$

Notice that the phase-amplitude distribution satisfies $P(i) \geqslant 0$ for any $i$ and $\sum_{j=1}^{n} P(j)=1$, akin to a probability mass function. The intensity of PAC can be inferred intuitively by visual inspection of the plot of $P$ over all the bins. Nevertheless, to provide a quantitative measure of PAC intensity, the $\mathrm{MI}$ is defined as

$$
\mathrm{MI}=\frac{D_{K L}(P, U)}{\log (n)},
$$

where $\quad D_{K L}(P, U)=\log (n)-H(P), \quad H(P)=$ $-\sum_{j=1}^{n} P(j) \log [P(j)]$, and $U$ represent the uniform distribution. Loosely speaking, the MI is a scaled Kullback-Leibler distance of the phase-amplitude distribution from the uniform distribution, satisfying $0 \leqslant \mathrm{MI} \leqslant 1$. Note that the Kullback-Leibler distance is widely used in information theory and statistics to measure the distance between two distributions. $\mathrm{MI}=1$ means that $P$ is a Dirac-like distribution, while $\mathrm{MI}=0$ is equivalent to a uniform distribution. Thus, for any signal, a larger MI means more intense PAC.

\section{PLV}

Given two time series of phases $\phi_{1}(t)$ and $\phi_{2}(t)$, the PLV is computed using the following formula:

$$
\operatorname{PLV}=\frac{1}{T}\left|\sum_{t=1}^{T} e^{\mathrm{i}\left[\phi_{1}(t)-\phi_{2}(t)\right]}\right|,
$$

where $T$ is the number of sample time points. The PLV takes value on $[0,1]$, where 1 reflects that the two phases are locked and 0 means that they are completely desynchronized. For a real signal, its time series of phases can be computed using the Hilbert transform.

In our case, we use PLV to measure the correlation of the fast amplitudes between regions. Let $\bar{r}_{\mathrm{f}}^{1}(t)$ and $\bar{r}_{\mathrm{f}}^{2}(t)$ be the average amplitudes in the two regions. Using the Hilbert transform, the series of phases, denoted by $\Phi\left(\bar{r}_{\mathrm{f}}^{1}\right)$ and $\Phi\left(\bar{r}_{\mathrm{f}}^{2}\right)$, can be computed, respectively. Therefore, the PLV value of $\Phi\left(\bar{r}_{\mathrm{f}}^{1}\right)$ and $\Phi\left(\bar{r}_{\mathrm{f}}^{2}\right)$ can be obtained by Eq. (A1). A large PLV means that the two fast amplitudes are highly correlated, while PLV $=0$ implies that they are uncorrelated.
[1] A. Arenas, A. Díaz-Guilera, J. Kurths, Y. Moreno, and C. Zhou, Synchronization in complex networks, Phys. Rep. 469, 93 (2008).

[2] R. T. Canolty, E. Edwards, S. S. Dalal, M. Soltani, S. S. Nagarajan, H. E. Kirsch, M. S. Berger, N. M. Barbaro, and R. T. Knight, High gamma power is phase-locked to theta oscillations in human neocortex, Science 313, 1626 (2006).

[3] O. Jensen and L. L. Colgin, Cross-frequency coupling between neuronal oscillations, Trends Cogn. Sci. 11, 267 (2007).

[4] R. T. Canolty and R. T. Knight, The functional role of crossfrequency coupling, Trends Cogn. Sci. 14, 506 (2010).

[5] B. Voloh, T. A. Valiante, S. Everling, and T. Womelsdorf, Theta-gamma coordination between anterior cingulate and pre- frontal cortex indexes correct attention shifts, Proc. Natl. Acad. Sci. USA 112, 8457 (2015).

[6] M. X. Cohen, N. Axmacher, D. Lenartz, C. E. Elger, V. Sturm, and T. E. Schlaepfer, Good vibrations: Cross-frequency coupling in the human nucleus accumbens during reward processing, J. Cognit. Neurosci. 21, 875 (2009).

[7] N. Axmacher, M. M. Henseler, O. Jensen, I. Weinreich, C. E. Elger, and J. Fell, Cross-frequency coupling supports multiitem working memory in the human hippocampus, Proc. Natl. Acad. Sci. USA 107, 3228 (2010).

[8] T. Yanagisawa, O. Yamashita, M. Hirata, H. Kishima, Y. Saitoh, T. Goto, T. Yoshimine, and Y. Kamitani, Regulation of motor 
representation by phase-amplitude coupling in the sensorimotor cortex, J. Neurosci. 32, 15467 (2012).

[9] F. Roux and P. J. Uhlhaas, Working memory and neural oscillations: alpha-gamma versus theta-gamma codes for distinct wm information? Trends Cogn. Sci. 18, 16 (2014).

[10] P. Lakatos, G. Karmos, A. D. Mehta, I. Ulbert, and C. E. Schroeder, Entrainment of neuronal oscillations as a mechanism of attentional selection, Science 320, 110 (2008).

[11] A. B. Tort, R. W. Komorowski, J. R. Manns, N. J. Kopell, and $\mathrm{H}$. Eichenbaum, Theta-gamma coupling increases during the learning of item-context associations, Proc. Natl. Acad. Sci. USA 106, 20942 (2009).

[12] C. von Nicolai, G. Engler, A. Sharott, A. K. Engel, C. K. Moll, and M. Siegel, Corticostriatal coordination through coherent phase-amplitude coupling, J. Neurosci. 34, 5938 (2014).

[13] T. Menara, G. Baggio, D. S. Bassett, and F. Pasqualetti, Stability conditions for cluster synchronization in networks of heterogeneous kuramoto oscillators, IEEE Trans. Control. Netw. Syst. 7, $302(2020)$

[14] A. P. Millán, J. J. Torres, and G. Bianconi, Explosive HigherOrder Kuramoto Dynamics On Simplicial Complexes, Phys. Rev. Lett. 124, 218301 (2020).

[15] Y. Qin, M. Cao, B. D. O. Anderson, D. S. Bassett, and F. Pasqualetti, Mediated remote synchronization of kuramotosakaguchi oscillators: The number of mediators matters, IEEE Contr. Syst. Lett. 5, 767 (2021).

[16] T. Menara, G. Baggio, D. S. Bassett, and F. Pasqualetti, A framework to control functional connectivity in the human brain, in Proceedings of IEEE 58th Conference on Decision and Control (CDC) (IEEE, Piscataway, 2019), pp. 4697-4704.

[17] Y. Qin, Y. Kawano, O. Portoles, and M. Cao, Partial phase cohesiveness in networks of Kuramoto oscillator, IEEE Trans. Auto. Control (2021), doi: 10.1109/TAC.2021.3062005.

[18] D. Métivier, L. Wetzel, and S. Gupta, Onset of synchronization in networks of second-order Kuramoto oscillators with delayed coupling: Exact results and application to phase-locked loops, Phys. Rev. Res. 2, 023183 (2020).

[19] J. H. Meng and H. Riecke, Synchronization by uncorrelated noise: interacting rhythms in interconnected oscillator networks, Sci. Rep. 8, 6949 (2018).

[20] Z. G. Nicolaou, M. Sebek, I. Z. Kiss, and A. E. Motter, Coherent Dynamics Enhanced by Uncorrelated Noise, Phys. Rev. Lett. 125, 094101 (2020).

[21] J. D. Hart, Y. Zhang, R. Roy, and A. E. Motter, Topological Control of Synchronization Patterns: Trading Symmetry for Stability, Phys. Rev. Lett. 122, 058301 (2019).

[22] E. Ullner and A. Politi, Self-Sustained Irregular Activity in an Ensemble of Neural Oscillators, Phys. Rev. X 6, 011015 (2016).

[23] E. Montbrió and D. Pazó, Kuramoto Model for ExcitationInhibition-Based Oscillations, Phys. Rev. Lett. 120, 244101 (2018).

[24] Y. S. Perl, H. Bocaccio, I. Pérez-Ipiña, F. Zamberlán, J. Piccinini, H. Laufs, M. Kringelbach, G. Deco, and E. Tagliazucchi, Generative Embeddings of Brain Collective Dynamics Using Variational Autoencoders, Phys. Rev. Lett. 125, 238101 (2020).

[25] P. Wulff, A. A. Ponomarenko, M. Bartos, T. M. Korotkova, E. C. Fuchs, F. Bähner, M. Both, A. B. L. Tort, N. J. Kopell, W. Wisden et al., Hippocampal theta rhythm and its coupling with gamma oscillations require fast inhibition onto parvalbumin- positive interneurons, Proc. Natl. Acad. Sci. USA 106, 3561 (2009).

[26] L. Fontolan, M. Krupa, A. Hyafil, and B. Gutkin, Analytical insights on theta-gamma coupled neural oscillators, J. Math. Neurosci. 3, 16 (2013).

[27] T. Akam, I. Oren, L. Mantoan, E. Ferenczi, and D. M Kullmann, Oscillatory dynamics in the hippocampus support dentate gyrus-ca3 coupling, Nat. Neurosci. 15, 763 (2012).

[28] A. C. Onslow, M. W. Jones, and R. Bogacz, A canonical circuit for generating phase-amplitude coupling, PLoS One 9, e102591 (2014).

[29] M. Jedynak, A. J. Pons, and J. Garcia-Ojalvo, Cross-frequency transfer in a stochastically driven mesoscopic neuronal model, Front. Comput. Neurosci. 9, 14 (2015).

[30] M. Chehelcheraghi, C. van Leeuwen, E. Steur, and C. Nakatani, A neural mass model of cross frequency coupling, PloS One $\mathbf{1 2}$ e0173776 (2017).

[31] M. Chehelcheraghi, C. Nakatani, E. Steur, and C. van Leeuwen, A neural mass model of phase-amplitude coupling, Biol. Cybern. 110, 171 (2016).

[32] A. Hyafil, A.-L. Giraud, L. Fontolan, and B. Gutkin, Neural cross-frequency coupling: Connecting architectures, mechanisms, and functions, Trends Neurosci. 38, 725 (2015).

[33] A. Von Stein and J. Sarnthein, Different frequencies for different scales of cortical integration: from local gamma to long range alpha/theta synchronization, Int. J. Psychophysiol. 38, 301 (2000).

[34] A. Salova and R. M. D'Souza, Decoupled synchronized states in networks of linearly coupled limit cycle oscillators, Phys. Rev. Research 2, 043261 (2020).

[35] M. H. Matheny, J. Emenheiser, W. Fon, A. Chapman, A. Salova, M. Rohden, J. Li, M. H. de Badyn, M. Pósfai, L. Duenas-Osorio et al., Exotic states in a simple network of nanoelectromechanical oscillators, Science 363, eaav7932 (2019).

[36] T. Nishikawa and A. E. Motter, Symmetric States Requiring System Asymmetry, Phys. Rev. Lett. 117, 114101 (2016).

[37] A. Zakharova, M. Kapeller, and E. Schöll, Chimera Death: Symmetry Breaking in Dynamical Networks, Phys. Rev. Lett. 112, 154101 (2014).

[38] C. R. Laing, Chimeras in networks of planar oscillators, Phys. Rev. E 81, 066221 (2010).

[39] N. Brunel and V. Hakim, Fast global oscillations in networks of integrate-and-fire neurons with low firing rates, Neural Comput. 11, 1621 (1999).

[40] H. G. Schuster and P. Wagner, A model for neuronal oscillations in the visual cortex, Biol. Cybern. 64, 77 (1990).

[41] G. Deco, E. Tagliazucchi, H. Laufs, A. Sanjuán, and M. L. Kringelbach, Novel intrinsic ignition method measuring localglobal integration characterizes wakefulness and deep sleep, Eneuro 4, ENEURO.0106-17.2017 (2017).

[42] M. L. Kringelbach, A. R. McIntosh, P. Ritter, V. K. Jirsa, and G. Deco, The rediscovery of slowness: Exploring the timing of cognition, Trends Cogn. Sci. 19, 616 (2015).

[43] C. Börgers and N. Kopell, Effects of noisy drive on rhythms in networks of excitatory and inhibitory neurons, Neural Comput. 17, 557 (2005).

[44] A. B. Tort, M. A. Kramer, C. Thorn, D. J. Gibson, Y. Kubota, A. M. Graybiel, and N. J. Kopell, Dynamic cross-frequency 
couplings of local field potential oscillations in rat striatum and hippocampus during performance of a t-maze task, Proc. Natl. Acad. Sci. USA 105, 20517 (2008).

[45] A. Sirota, S. Montgomery, S. Fujisawa, Y. Isomura, M. Zugaro, and G. Buzsáki, Entrainment of neocortical neurons and gamma oscillations by the hippocampal theta rhythm, Neuron 60, 683 (2008).

[46] A. Mazzoni, S. Panzeri, N. K. Logothetis, and N. Brunel, Encoding of naturalistic stimuli by local field potential spectra in networks of excitatory and inhibitory neurons, PLoS Comput. Biol. 4, e1000239 (2008).

[47] C. S. Zandvoort and G. Nolte, Defining the filter parameters for phase-amplitude coupling from a bispectral point of view, J. Neurosci. Meth. 350, 109032 (2021).

[48] M. Provansal, C. Mathis, and L. Boyer, Bénard-von kármán instability: Transient and forced regimes, J. Fluid Mech. 182, 1 (1987).

[49] A. B. L. Tort, R. Komorowski, H. Eichenbaum, and N. Kopell, Measuring phase-amplitude coupling between neuronal oscillations of different frequencies, J. Neurophysiol. 104, 1195 (2010).
[50] J. A. White, M. I. Banks, R. A. Pearce, and N. J. Kopell, Networks of interneurons with fast and slow $\gamma$-aminobutyric acid type A (GABAA) kinetics provide substrate for mixed gamma-theta rhythm, Proc. Natl. Acad. Sci. USA 97, 8128 (2000).

[51] M. A. Whittington and R. D. Traub, Interneuron diversity series: Inhibitory interneurons and network oscillations in vitro, Trends Neurosci. 26, 676 (2003).

[52] A. Hyafil, L. Fontolan, C. Kabdebon, B. Gutkin, and A.-L. Giraud, Speech encoding by coupled cortical theta and gamma oscillations, Elife 4, e06213 (2015).

[53] J. A. Sanders, F. Verhulst, and J. Murdock, Averaging Methods in Nonlinear Dynamical Systems (Springer, 2007), Vol. 59.

[54] G. Nolte, A. Ziehe, V. V. Nikulin, A. Schlögl, N. Krämer, T. Brismar, and K.-R. Müller, Robustly Estimating The Flow Direction of Information in Complex Physical Systems, Phys. Rev. Lett. 100, 234101 (2008).

[55] J.-Y. Moon, U. Lee, S. Blain-Moraes, and G. A. Mashour, General relationship of global topology, local dynamics, and directionality in large-scale brain networks, PLoS Comput. Biol. 11, e1004225 (2015). 